\title{
A time-domain control signal detection technique for OFDM
}

\author{
Saheed A. Adegbite ${ }^{*}$, Scott G. McMeekin and Brian G. Stewart ${ }^{\dagger}$
}

\begin{abstract}
Transmission of system-critical control information plays a key role in efficient management of limited wireless network resources and successful reception of payload data information. This paper uses an orthogonal frequency division multiplexing (OFDM) architecture to investigate the detection performance of a time-domain approach used to detect deterministic control signalling information. It considers a type of control information chosen from a finite set of information, which is known at both transmitting and receiving wireless terminals. Unlike the maximum likelihood (ML) estimation method, which is often used, the time-domain detection technique requires no channel estimation and no pilots as it uses a form of time-domain correlation as the means of detection. Results show that when compared with the ML method, the time-domain approach improves detection performance even in the presence of synchronisation error caused by carrier frequency offset.
\end{abstract}

Keywords: Carrier frequency offset (CFO), Channel estimation, Selective control information, Maximum likelihood $(\mathrm{ML})$, Orthogonal frequency division multiplexing (OFDM), Time-domain correlation

\section{Introduction}

Efficient management of wireless network resources is essential in wireless communication systems. In most wireless systems, the management and allocation of system resources is usually coordinated through the use of some control signalling information [1]. Such control information carries important signalling information from a transmitting device (a base station) to a receiving terminal (a mobile station). In general, control information carries system-critical information about network configuration in order to enable successful reception of payload data and to facilitate efficient management of limited network resources [1-3]. To this end, the receiver must be able to successfully determine the transmitted control information for subsequent use during decoding of the payload data.

In wireless systems, the transmitted information usually consists of two components: control information and payload user data. The control information may be chosen from a finite set of known and pre-determined candidate information and can also be the same for multiple users.

\footnotetext{
*Correspondence: sa.adegbite@gmail.com

†Equal contributors

School of Engineering and Built Environment, Glasgow Caledonian University,

70 Cowcaddens Road, G4 OBA, Glasgow, UK
}

Unlike the control information, the payload (user data) is randomly generated at the transmitter and it is usually different for each user. In terms of data recovery, detection of control signalling information is entirely different from the decoding procedures of payload user data information. In the literature, payload user-data information is normally recovered through what is generally considered as one-tap equalization (symbol-by-symbol) decoding where each subcarrier symbol is independently processed during data decoding [4-6]. On the other hand, a block-level detection is the most appropriate decoding approach for control information, particularly, selective control information (SCI) [7], which is considered in this paper. In a block-level decoding procedure, it is usually assumed that the SCI is represented by a group of subcarriers, which is chosen from a fixed number of pre-encoded sets of possible SCI (known at both transmitting and receiving terminals), as described in [7-10]. Therefore, at the receiver, the received group of subcarriers used to represent the SCI is collectively used during decoding [7]. For instance, a form of SCI can be used to represent the type of modulation scheme of payload user-data information. As an example, using the well-known quadrature 
amplitude modulation (QAM) scheme, payload information may be modulated using 4-QAM, 16-QAM, 64-QAM or even 256-QAM. To recover the payload information, the receiver must first determine the type of modulation scheme. This can be achieved by encoding each possible type of modulation with a pre-coded information on a number of subcarrier to facilitate subsequent recovery through some form of block-level decoding.

Using a baseband orthogonal frequency division multiplexing (OFDM) architecture, this paper studies block-level detection of SCI. An OFDM architecture is considered because it is the adopted physical layer technology in several high-speed wireless systems such as Long-Term Evolution (LTE) [11-13]. Another example of SCI used within LTE systems is the control format indicator (CFI), which carries key system information that enables each user equipment (UE) to correctly decode the main control information within the LTE physical downlink control channel [1]. A detailed description of CFI and other forms of LTE control information can be found in [14].

To recover transmitted SCI, a maximum likelihood (ML) criterion is often used as the standard detection approach [7-10]. Unfortunately, the ML estimation scheme normally requires some form of channel mitigation through the use of, for example, pilot-assisted channel estimation in order to improve decoding performance [8]. As a consequence, transmission of additional system resources in the form of pilot or training signals is required to determine the channel response. However, to improve channel estimation, particularly in severe fading channel environments, a large number of pilot signals is required, which reduces the overall spectral efficiency [15].

To address the practical challenges associated with the ML-based receiver, this paper investigates the use of a time-domain approach for the recovery of SCI. The timedomain detection technique uses a decision rule that is derived from a time-domain correlation between the transmitted and received SCI, and as a consequence, eliminates the need for channel estimation at the receiver. Using the block error rate (BLER) performance metric, simulations evaluate the detection performance of the ML and the time-domain estimation methods in the presence of synchronisation error due to carrier frequency offset (CFO). Note that no CFO compensation is performed in this paper. Examples of recent studies on $\mathrm{CFO}$ and inter-carrier interference (ICI) (Intercarrier inteference) compensation are presented in [16] and [17].

The paper is structured as follows. Section 2 outlines the considered baseband OFDM architecture and describes an ML-based receiver to recover the transmitted SCI. Section 3 describes the implementation of the time-domain detection technique. Section 4 presents the simulation results and related discussions on the BLER performance of the two considered methods. Finally, Section 5 highlights the main contributions of the paper.

\section{System model}

This section describes the baseband OFDM architecture used for the investigations within this paper. It also gives an overview of the ML-based receiver studied in, for example, [7].

\subsection{Transmitter}

Let $X$ be an OFDM sequence of length $N_{\mathrm{v}}$, which is the raw input for the inverse fast Fourier transform (IFFT) stage before zero padding. Then, for $0 \leq k \leq N_{\mathrm{v}}-1$ where $k$ represent a subcarrier index, $\boldsymbol{X}$ is written as

$$
\boldsymbol{X}=\left[\boldsymbol{X}[0] \boldsymbol{X}[1] \boldsymbol{X}[k] \ldots X\left[N_{\mathrm{v}}-1\right]\right] .
$$

For simplicity, it is assumed $X$ consists of randomly generated data, $\boldsymbol{X}_{\boldsymbol{d}}$ of size $N_{d}$, a pilot sequence, $\boldsymbol{X}_{\boldsymbol{p}}$ of size $N_{p}$, and an SCI vector, $\boldsymbol{X}_{\boldsymbol{c}}$ of size $N_{c}$, such that $N_{v}=$ $N_{p}+N_{c}+N_{d}$. Each element of $\boldsymbol{X}$ is mapped to a subcarrier symbol $\boldsymbol{X}[k]$ through, for example, a subcarrier mapping scheme described as follows:

1. First, each pilot subcarrier symbol is inserted at every $L$ subcarrier indices where $L$ defines the pilot spacing. In this paper, $L$ is set to 6 , so that the pilots are mapped to, for example, $k=\{0,6,12,18, \ldots\}$, as specified for LTE systems [18].

2. Given that $\boldsymbol{X}_{\boldsymbol{c}} \in \mathcal{S}$ where $\mathcal{S}$ consists of $U$ candidate sequence vectors where $\mathcal{S}$ is considered to be deterministic and is known at both transmitter and the receiver. For $1 \leq u \leq U$, each sequence vector in $\mathcal{S}$ is denoted by $\boldsymbol{S}_{u}$, i.e.,

$$
\mathcal{S}=\left\{S_{1}, S_{2}, S_{u} \ldots S_{U}\right\} .
$$

For an arbitrary index $c$ where $0 \leq c \leq N_{c}-1$, each SCI $\boldsymbol{S}_{u}$ is

$$
\boldsymbol{S}_{u}=\left[\boldsymbol{S}_{u}[0], \boldsymbol{S}_{u}[1], \boldsymbol{S}_{u}[c] \ldots \boldsymbol{S}_{u}\left[N_{c}-1\right]\right]
$$

As in most practical applications, each $\boldsymbol{S}_{u}[c]$ is QPSK modulated. Hence, the magnitude of $\boldsymbol{S}_{u}[c]$ is 1 . Let $\bar{u}$ define the $u$ index of the transmitted sequence chosen from $\mathcal{S}$. Hence,

$$
X_{\boldsymbol{c}}=S_{\bar{u}} \text { where } S_{\bar{u}} \in \mathcal{S} .
$$

Each element of $\boldsymbol{X}_{\boldsymbol{c}}$ is mapped to the next available $N_{c}$ subcarriers in $\boldsymbol{X}$. It can be noted from (3) that transmitted SCI can be known using the index $\bar{u}$. 
3. Finally, the remaining $N_{d}$ un-allocated subcarriers in $\boldsymbol{X}$ are assigned to each element of randomly generated modulated data $\boldsymbol{X}_{\boldsymbol{d}}$.

Figure 1 shows the block diagram representation of a baseband OFDM transmitter architecture used for SCI transmission. It can be noted that this architecture inserts some pilots to enable channel estimation as required by the ML estimation method. However, as will be shown later, these pilots are not used and remain passive in the time-domain detection method.

As in the standard OFDM transmitter, the OFDM sequence, $X$ is transformed into a time-domain OFDM signal, $\boldsymbol{x}$ of length $N$, using an $N$-point IFFT where $N>$ $N_{v}$, i.e. zero padding is applied during IFFT where $N-N_{v}$ zeros are appended after the first $N_{\nu}$ subcarriers. For $0 \leq$ $n \leq N-1$, each individual sample $\boldsymbol{x}[n]$ in $\boldsymbol{x}$ is obtained through

$$
\begin{aligned}
\boldsymbol{x}[n] & =\frac{1}{N} \sum_{k=0}^{N_{\mathrm{v}}-1} \boldsymbol{X}[k] \exp (j 2 \pi n k / N) \\
& =\underset{N-\text { point }}{\operatorname{IFFT}}\{\boldsymbol{X}\},
\end{aligned}
$$

where IFFT $\{\cdot\}$ denotes an IFFT function.

Similarly, as in the standard OFDM described in, for example, [19], the length of the OFDM signal $x$ is further extended by a cyclic prefix (CP) so as to mitigate channel fading and inter-symbol interference (ISI) [20].

\subsection{Receiver}

At the receiver, $\mathrm{CP}$ samples are first removed. After the fast fourier transform (FFT) stage at the receiver, the frequency domain representation of the received OFDM symbol $\boldsymbol{Y}$ is given as [21]

$$
Y=H X+V
$$

Note that $\boldsymbol{H}$ and $\boldsymbol{V}$ in (5) are, respectively, the frequency domain representation of the complex-valued channel frequency response and additive white Gaussian noise (AWGN). Hence, each subcarrier $\boldsymbol{Y}[k]$ in $\boldsymbol{Y}$ is written as

$$
\boldsymbol{Y}[k]=\boldsymbol{H}[k] \boldsymbol{X}[k]+\boldsymbol{V}[k] .
$$

Let $f_{0}$ and $\Delta f$, respectively, represent the frequency offset and the OFDM subcarrier spacing (both in Hertz). Then, a normalised CFO, $\varepsilon$ is expressed by [21]

$$
\varepsilon=f_{0} / \Delta f
$$

In the presence of CFO, $\boldsymbol{Y}$ is represented by [21]

$$
\boldsymbol{Y}=\boldsymbol{G}(\varepsilon) \boldsymbol{H} \boldsymbol{X}+\boldsymbol{V},
$$

where

$$
\boldsymbol{G}(\varepsilon)=\boldsymbol{F} \boldsymbol{C}[\varepsilon] \boldsymbol{F}^{\mathrm{H}} .
$$

The term $\boldsymbol{F}$ in (9) represents an $N \times N$ matrix with each element $\boldsymbol{F}_{n k}$ given by [22]

$$
\boldsymbol{F}_{n k}=\exp (-j 2 \pi n k / N) / \sqrt{N}
$$

and $C[\varepsilon]$ in (9) is a diagonal matrix expressed by [21]

$$
C[\varepsilon]=\left(\begin{array}{cccc}
1 & 0 & \cdots & 0 \\
0 & \exp (j 2 \pi \varepsilon / N) & \cdots & 0 \\
\vdots & \vdots & \ddots & \vdots \\
0 & 0 & \cdots & \exp (j 2 \pi \varepsilon(N-1) / N)
\end{array}\right)
$$

Similar to (5), the received SCI sequence vector, $Y_{c}$, is given by

$$
Y_{c}=H_{c} X_{c}+V_{c},
$$

where $\boldsymbol{H}_{\boldsymbol{c}}$ and $\boldsymbol{V}_{\boldsymbol{c}}$, respectively, represent the sub-channel gains and AWGN components associated with the received selective sequence $\boldsymbol{Y}_{\boldsymbol{c}}$. Similarly, the received pilot, $\boldsymbol{Y}_{\boldsymbol{p}}$, is given by

$$
Y_{p}=H_{p} X_{p}+V_{p}
$$

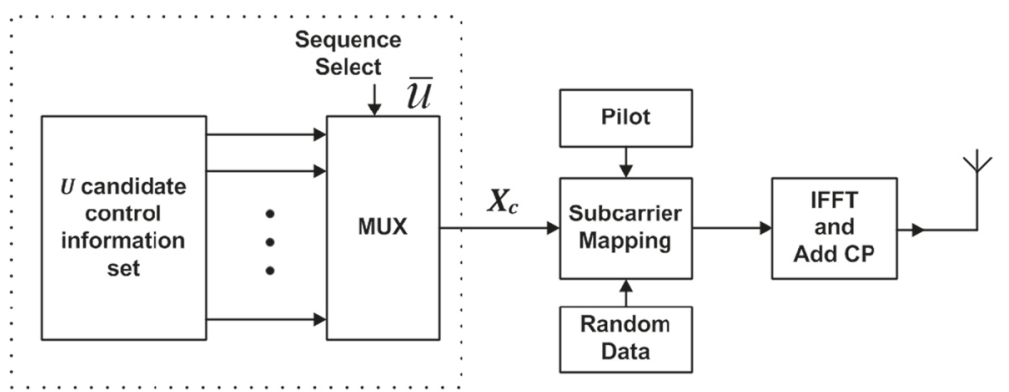

Fig. 1 A block diagram representation of a baseband OFDM transmitter for $\mathrm{SCl}$ transmission 
where $H_{p}$ and $V_{p}$, respectively, represent the sub-channel gain and AWGN components within the received pilot sequence $\boldsymbol{Y}_{\boldsymbol{p}}$.

Thus, to recover $\boldsymbol{X}_{\boldsymbol{c}}$, the receiver must determine an estimate of $\bar{u}$ since the transmitted SCI is chosen from a deterministic set, $\mathcal{S}$, and is considered to be known at the receiver.

\subsection{ML-based detection}

Let $\hat{u}$ denote an estimate of $\bar{u}$. Then, using the ML estimation criterion, $\hat{u}$ is obtained from [7]

$$
\hat{u}=\underset{u}{\arg \min }\left\|\boldsymbol{Y}_{\boldsymbol{c}}-\hat{\boldsymbol{H}}_{\boldsymbol{c}} \boldsymbol{S}_{u}\right\|^{2}
$$

The term $\hat{\boldsymbol{H}}_{\boldsymbol{c}}$ represents the sub-channel estimates (associated with the received SCI). In this paper, $\hat{\boldsymbol{H}}_{\boldsymbol{c}}$ is obtained from a linear interpolation between the least squares (LS) sub-channel pilot estimates, $\hat{H}_{p}$, where

$$
\hat{H}_{p}=Y_{p} / X_{p}
$$

In (14), it can be seen that the ML estimation criterion is a form of Euclidean distance minimisation function. Again, from (14), it can be noted that the detection performance of the ML estimation method depends mainly on the channel estimate $\hat{\boldsymbol{H}}_{\boldsymbol{c}}$. Hence, poor channel estimation is expected to result in erroneous estimate of $\hat{u}$. This is the main drawback of the ML estimation method.

\section{The proposed method}

In a classical channel estimation scheme such as minimum mean square error (MMSE), some form of correlation is often applied to determine the channel response $[4,23]$.
In addition, studies from, for example, [24] and [25] suggest that a time-domain approach is more robust against severe ISI compared with the frequency-domain method of channel equalization. This was partly due to the partial elimination of the channel tap with the most energy in the time-domain [26]. These principles form the basis behind the use of the time-domain estimation technique considered in this paper.

In the time-domain detection scheme, some form of correlation function is derived from the 'correlation' of $Y_{\boldsymbol{c}}$ with each of the candidate sequences within the set $\mathcal{S}$ since $\mathcal{S}$ is deterministic and known at both transmitting and receiving terminals. The rationale for the use of a time-domain correlation follows a general principle of deterministic signal correlation, which suggests that similar to the auto-correlation principle, there exists an inherent correlation between $Y_{c}$ and $X_{c}$, in the presence of limited noise levels, since $\boldsymbol{X}_{\boldsymbol{c}}$ is deterministic and chosen from, for example, a known set $\mathcal{S}$.

Figure 2 shows the block diagram representation of a baseband OFDM receiver for SCI detection based on the ML and the time-domain estimation methods. As previously stated, it can be noted from Fig. 2 that the time-domain method requires no channel estimation. The time-domain decision rule is now described through the use of the well-known discrete correlation theorem (DCT) (Discrete correlation theorem).

\subsection{Time-domain decision criterion}

Using the DCT, a correlation function (CORR) of two arbitrary time-domain signals $\boldsymbol{c}_{\mathbf{1}}$ and $\boldsymbol{c}_{\mathbf{2}}$ (of the same size) is obtained from [27]

$$
\operatorname{CORR}\left\{c_{1}, c_{2}\right\}=\operatorname{IFFT}\left\{C_{1} \times C_{2}^{*}\right\}
$$
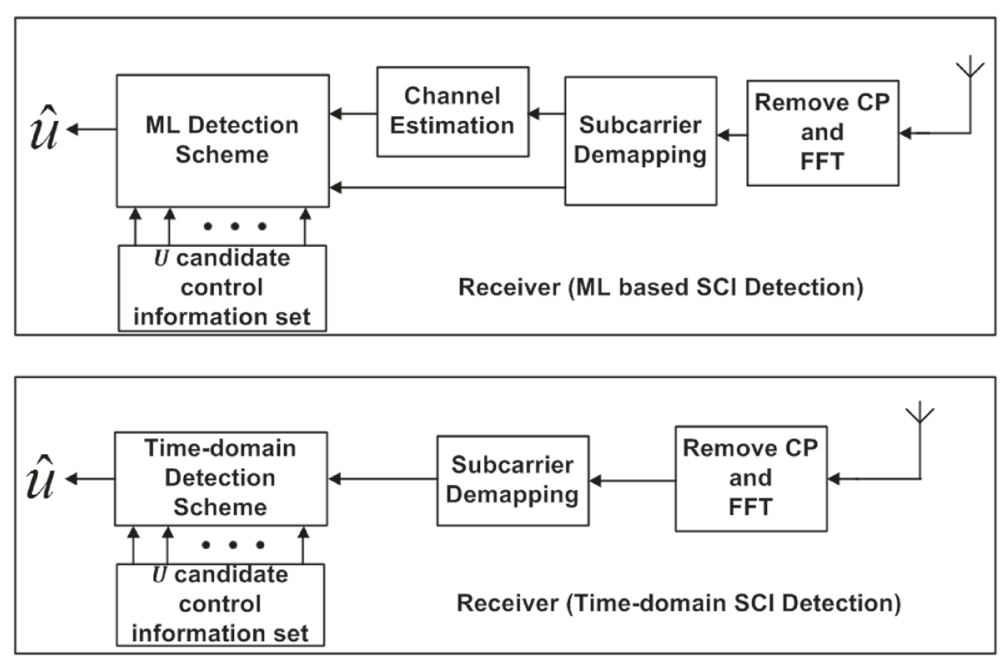

Fig. 2 A block diagram representation of a baseband OFDM receiver for SCl detection using ML and the time-domain method 
where ${ }^{*}$ represents the complex conjugation and $C_{\mathbf{1}}$ and $C_{2}$ are, respectively, the frequency domain representations of $c_{1}$ and $c_{2}$, i.e.

$$
\begin{aligned}
& C_{1}=\operatorname{FFT}\left\{c_{1}\right\} \\
& C_{2}=\operatorname{FFT}\left\{c_{2}\right\},
\end{aligned}
$$

where FFT $\{\cdot\}$ denotes FFT function.

Similar to the definition in (16), a term $Z_{u}$ is obtained from direct multiplication of $\boldsymbol{Y}_{\boldsymbol{c}}$ with the complex conjugate of each $\boldsymbol{S}_{u}$. Thus, $\boldsymbol{Z}_{u}$ is derived from

$$
\begin{aligned}
\boldsymbol{Z}_{u} & =\boldsymbol{Y}_{\boldsymbol{c}} \times \boldsymbol{S}^{*}{ }_{u} \\
& =\left(\boldsymbol{H}_{\boldsymbol{c}} \boldsymbol{X}_{\boldsymbol{c}}+\boldsymbol{V}_{\boldsymbol{c}}\right) \times \boldsymbol{S}^{*}{ }_{u} \\
& =\left(\boldsymbol{H}_{\boldsymbol{c}} \boldsymbol{X}_{\boldsymbol{c}} \boldsymbol{S}^{*}{ }_{u}\right)+\left(\boldsymbol{V}_{\boldsymbol{c}} \boldsymbol{S}^{*}{ }_{u}\right) \\
& =\left(\frac{\boldsymbol{H}_{\boldsymbol{c}} \boldsymbol{X}_{\boldsymbol{c}}}{\boldsymbol{S}_{u}}\right)+\left(\frac{\boldsymbol{V}_{\boldsymbol{c}}}{\boldsymbol{S}_{u}}\right) \\
& =\left(\frac{\boldsymbol{H}_{\boldsymbol{c}} \boldsymbol{X}_{\boldsymbol{c}}}{\boldsymbol{S}_{u}}\right)+\boldsymbol{V}_{\boldsymbol{c}, u}^{\prime} .
\end{aligned}
$$

For $0 \leq c \leq N_{c}-1, Z_{u}$ may be represented as

$$
Z_{u}=\left[Z_{u}[0], Z_{u}[1], Z_{u}[c] \ldots Z_{u}\left[N_{c}-1\right]\right] .
$$

Alternatively, $\boldsymbol{Z}_{u}$ can also be written as

$$
\boldsymbol{Z}_{u}=\left\{\begin{array}{l}
\boldsymbol{H}_{\boldsymbol{c}}+\boldsymbol{V}_{\boldsymbol{c}, u}^{\prime}, \quad u=\bar{u}\left(\boldsymbol{X}_{\boldsymbol{c}}=\boldsymbol{S}_{u}\right) \\
\boldsymbol{H}_{\boldsymbol{c}} \boldsymbol{X}_{\boldsymbol{c}} \boldsymbol{S}^{*}{ }_{u}+\boldsymbol{V}_{\boldsymbol{c}, u}^{\prime},
\end{array}\right.
$$

By omitting the noise terms in (20) for simplicity (at high signal-to-noise ratio (SNR)), the expression for $Z_{u}$ in (20) is reduced to

$$
Z_{u} \approx \begin{cases}\boldsymbol{H}_{\boldsymbol{c}}, & u=\bar{u} \\ \boldsymbol{H}_{\boldsymbol{c}} \boldsymbol{X}_{\boldsymbol{c}} \boldsymbol{S}^{*}{ }_{u}, & \text { otherwise, }\end{cases}
$$

where $X_{c} S^{*}{ }_{u}$ is a complex-valued number with unity magnitude since each $S_{\boldsymbol{u}}$ is assumed to have the same magnitude.

From the expression in (20), it can be seen that the same channel term $\boldsymbol{H}_{\boldsymbol{c}}$ and identical noise term $V_{\boldsymbol{c}, u}^{\prime}$ is present in both $\boldsymbol{Z}_{u=\bar{u}}$ and $\boldsymbol{Z}_{u \neq \bar{u}}$ terms when $u=\bar{u}$ and $u \neq \bar{u}$, respectively. Hence, the term $\boldsymbol{H}_{\boldsymbol{c}}$ is considered (for decoding purpose) as a gain effect. Thus, without loss of generality, the main difference between each value of $\boldsymbol{Z}_{u=\bar{u}}$ and $\boldsymbol{Z}_{u \neq \bar{u}}$ can be written as

$$
Z_{u}[c] \approx \begin{cases}1, & u=\bar{u} \\ X_{c} S^{*}{ }_{u}, & \text { otherwise. }\end{cases}
$$

Similar to (16), a real-valued time-domain correlation function, $W_{u}$ is computed from $Z_{u}[c]$ using a $\mathcal{W}$-point IFFT where $\mathcal{W}$ must be a power of 2 and $\mathcal{W} \geq N_{c}$, i.e. $\mathcal{W}=N_{c}$ if $N_{c}$ is a power of 2; otherwise, $\mathcal{W}>N_{c}$.

For $0 \leq w \leq \mathcal{W}-1, W_{u}$ is written as

$$
\boldsymbol{W}_{u}=\left[\boldsymbol{W}_{u}[0], \boldsymbol{W}_{u}[1], \boldsymbol{W}_{u}[w] \ldots \boldsymbol{W}_{u}[\mathcal{W}-1]\right]
$$

where

$$
W_{u}=\left|\underset{\mathcal{W} \text {-point }}{\operatorname{IFFT}}\left\{Z_{u}\right\}\right|,
$$

where $|\cdot|$ computes the magnitude of a complex-valued variable. It should be noted that in this paper, the IFFT operation in (24) performs no zero padding since each considered value of $N_{c}$ is a power of 2, i.e. the $\mathcal{W}=N_{c}$. However, in cases (not shown in this paper) where $N_{c}$ is not a power of 2 , zero padding can be applied accordingly to reduce computational complexity and with no expected degradation in performance.

From the approximation of $\boldsymbol{Z}_{\bar{u}}$ in (22), the IFFT of $\boldsymbol{Z}_{\bar{u}}$ yields an impulse function. Hence, the value of $W_{\bar{u}}[w]$ (derived from $\boldsymbol{Z}_{\bar{u}}$ ) is approximated as

$$
W_{\bar{u}}[w]=\left\{\begin{array}{l}
1, w=0 \\
0,1 \leq w \leq N_{c}-1 .
\end{array}\right.
$$

Otherwise, $W_{u}[w]>0$ when $u \neq \bar{u}$.

Using the approximation in (25), it can be noted that the mean value of $\boldsymbol{W}_{\bar{u}}[w]$, i.e. $E\left\{\boldsymbol{W}_{\bar{u}}[w]\right\}$, is

$$
\begin{aligned}
E\left\{\boldsymbol{W}_{\bar{u}}[w]\right\} & =\frac{1}{\mathcal{W}} \sum_{w=0}^{\mathcal{W}-1} W_{\bar{u}}[w] \\
& \approx 1 / \mathcal{W}
\end{aligned}
$$

where $E$ is the expectation function.

Similarly, from the definition in (25), $E\left\{W_{u \neq \bar{u}}[w]\right\}$ is expected to be larger than $E\left\{\boldsymbol{W}_{\bar{u}}[w]\right\}$ because the corresponding values of $W_{u \neq \bar{u}}[w]$ are non-zero. Hence,

$$
E\left\{\boldsymbol{W}_{\bar{u}}[w]\right\} \ll E\left\{\boldsymbol{W}_{u \neq \bar{u}}[w]\right\} .
$$

Thus, in the presence of channel fading term $\boldsymbol{H}_{\boldsymbol{c}}$, the expression in (27) is still valid since the resulting timedomain functions $W_{\bar{u}}$ and $W_{u \neq \bar{u}}$ are both affected by the same channel component.

The expression in (27) therefore implies that an estimate of $\bar{u}$ corresponds to the $u$-index of the time-domain function with the minimum mean value amongst all $U$ 
Table 1 Power delay profile of the EVA multipath fading channel

\begin{tabular}{lccccccccc}
\hline Channel parameters & Tap 1 & Tap 2 & Tap 3 & Tap 4 & Tap 5 & Tap 6 & Tap 7 & Tap 8 & Tap 9 \\
\hline Path delay, ns & 0 & 30 & 150 & 310 & 370 & 710 & 1090 & 1730 & 2510 \\
Power, dB & 0.0 & -1.5 & -1.4 & -3.6 & -0.6 & -9.1 & -7.0 & -12.0 & -16.9 \\
\hline
\end{tabular}

time-domain functions. Hence, it can be said that the time-domain decision criterion minimises the mean value of the time-domain correlation function obtained from (24). Thus, the time-domain detection rule is defined as

$$
\hat{u}=\underset{u}{\arg \min } E\left\{\boldsymbol{W}_{u}[w]\right\} .
$$

From the expressions in (18) to (28), it can be noted that no channel estimation and no pilots are required in the time-domain method because the time-domain correlation inherently facilitates detection even in the presence of a fading channel [28]. However, as will be shown in the next section, one of the main limiting factors for the proposed detection method is the inaccuracy of the time-domain decision metric for small values of $N_{c}$.

\section{Simulation results}

This section presents the comparison of the detection performance of the two considered detection methods using the BLER metric.

\subsubsection{Computing BLER}

The BLER is not the same as the well-known bit error rate (BER) metric. In the case of BER, individual bit errors are considered. However, to compute the BLER, an error count between the actual value $\bar{u}$ that corresponds to the selected sequence $\boldsymbol{X}_{\boldsymbol{c}}$ and its estimate $\hat{u}$, obtained from the ML or the time-domain detection scheme, is evaluated. Hence, an erroneous block is when $\bar{u} \neq \hat{u}$; otherwise, there is a successful detection. For each SNR level, the BLER is evaluated as

$$
\mathrm{BLER}=\frac{1}{N_{\mathrm{BLK}}} \sum_{i=1}^{N_{\mathrm{BLK}}} \boldsymbol{R}_{i} .
$$

For $1 \leq i \leq N_{\mathrm{BLK}}$ where $N_{\mathrm{BLK}}$ is the number of OFDM symbol blocks (for a given SNR level), $\boldsymbol{R}_{i}$ is computed from

$$
\boldsymbol{R}_{i}= \begin{cases}1 & \text { if } \bar{u} \neq \hat{u} \\ 0 & \text { otherwise }\end{cases}
$$

\subsubsection{Simulation setup}

OFDM simulation considers transmission over a frequency-selective Rayleigh fading channel known as the extended vehicular type A (EVA), with a root mean square
(RMS) delay spread, $\tau_{r m s}$, of $357 \mathrm{~ns}$ [29]. Table 1 shows the power delay profile of the EVA channel [30]. Simulations use parameter values outlined in Table 2 and are carried out using MATLAB. To further understand the detection performance of each considered detection scheme, simulations also evaluate the BLER performance in the absence of a fading channel, i.e. the presence of AWGN only.

The BLER performance of the two considered methods is investigated as a function of $\varepsilon$ for each value of $N_{c}$. Note that since each chosen value of $N_{c}$ is a power of 2 , then $\mathcal{W}=N_{c}$. It should also be noted that for the ML scheme, simulations implement the pilot-assisted channel estimation procedures previously outlined in (15) and linear channel interpolation is used to obtain $\hat{\boldsymbol{H}}_{\boldsymbol{c}}$.

\subsubsection{Numerical results and discussions}

In the presence of only AWGN, Fig. 3a-d shows the BLER comparisons between the two considered detection methods with $N_{c}$ set to $8,16,32$ and 64 , respectively. Results in Fig. 3a show that for a small block size $\left(N_{c}=8\right)$, the ML scheme produces improved BLER performance compared with the time-domain method. However, results in Fig. 3b-d suggest that the detection performance of the time-domain estimation method is improved as $N_{c}$ is further increased. Results in Fig. 3 also demonstrate that in this case (i.e. with no fading channel effects), the BLER performance of the ML scheme is relatively the same even when $N_{c}$ is increased. In summary, results in Fig. 3 suggest that (1) the time-domain method is robust

Table 2 Simulation parameters

\begin{tabular}{ll}
\hline Parameters & Values \\
\hline OFDM subcarrier spacing & $15 \mathrm{KHz}$ \\
Sampling frequency & $3.84 \mathrm{MHz}$ \\
Pilot spacing, $L$ & 6 (i.e. $N_{p}=30$ ) \\
IFFT/FFT size, $N$ & 256 \\
OFDM sequence size, $N_{\text {sub }}$ & 180 \\
$N_{C}$ & $8,16,32$ and 64 \\
Guard interval (GI) & $5.21 \mu \mathrm{S}$ \\
Modulation & QPSK \\
$U$ & 4 \\
Number of OFDM symbol blocks, NBLK & 50,000 \\
Normalised CFO, $\varepsilon$ & $0,0.01$ and 0.05 \\
\hline
\end{tabular}



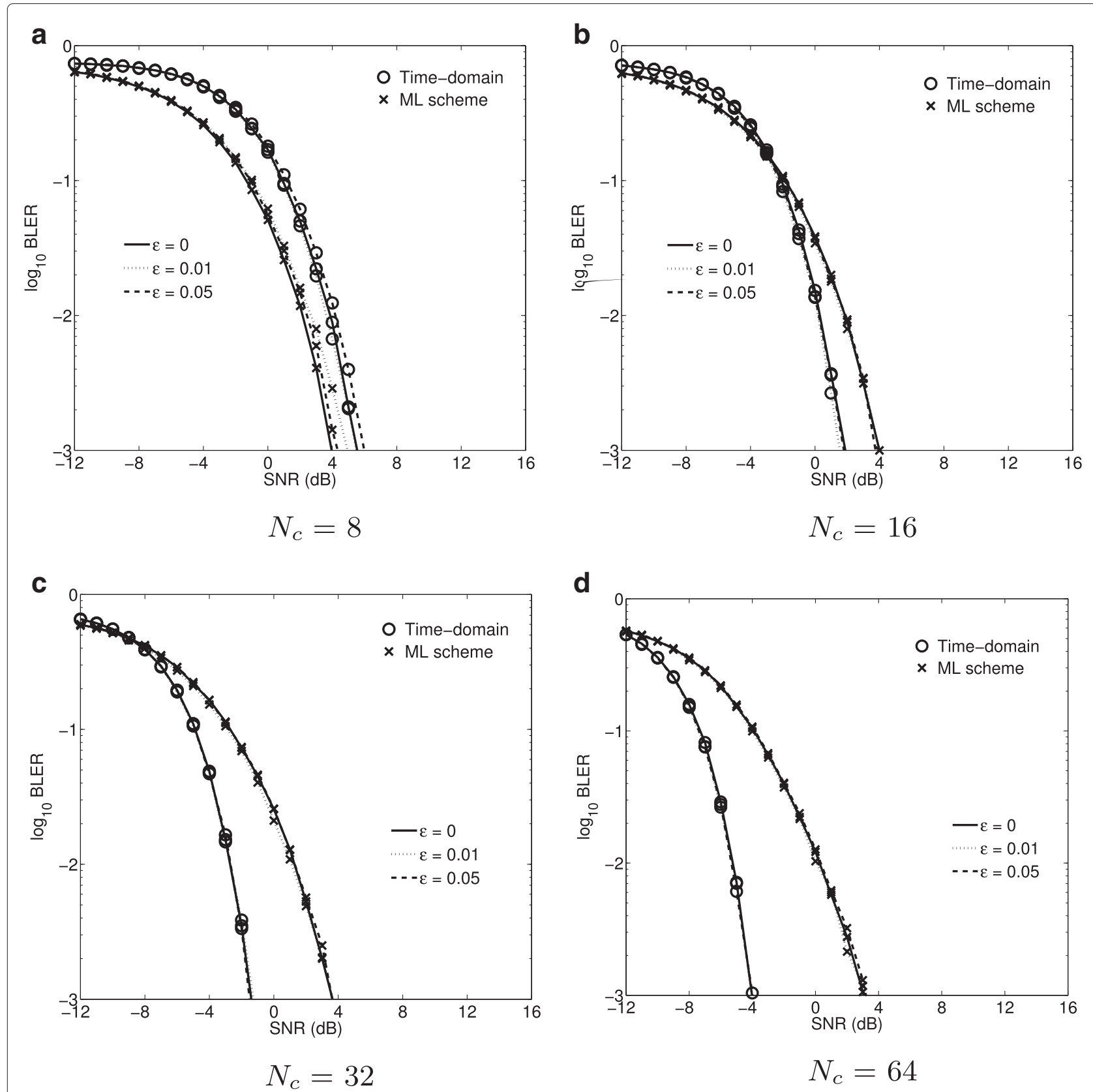

Fig. 3 BLER comparisons in the absence of channel fading

against synchronisation error due to CFO; (2) the detection performance of the time-domain method can be improved with larger values of $N_{c}$; and (3) in the absence of channel fading, the detection performance of the ML estimation method is nearly the same for all the considered values of $N_{c}$. Figure $4 \mathrm{a}-\mathrm{d}$ shows BLER comparisons when transmission is over the EVA fading channel. Similar to the previous results in Fig. 3a, results in Fig. 4a, b show that the ML estimation method outperforms the timedomain method when $N_{c}=8$ and 16 . However, as $N_{c}$ is further increased, the detection performance of the timedomain detection scheme is further improved compared with the ML detection approach, as shown in Fig. 4c, d. This is because as $N_{c}$ is further increased, statistical evaluation of the applied time-domain decision metric is more accurate, and as a result, its detection performance is improved compared with the ML scheme. Results further show that in the presence of frequency-selective channel fading effects, the ML scheme only produces minimal improvement in detection performance as $N_{c}$ is 


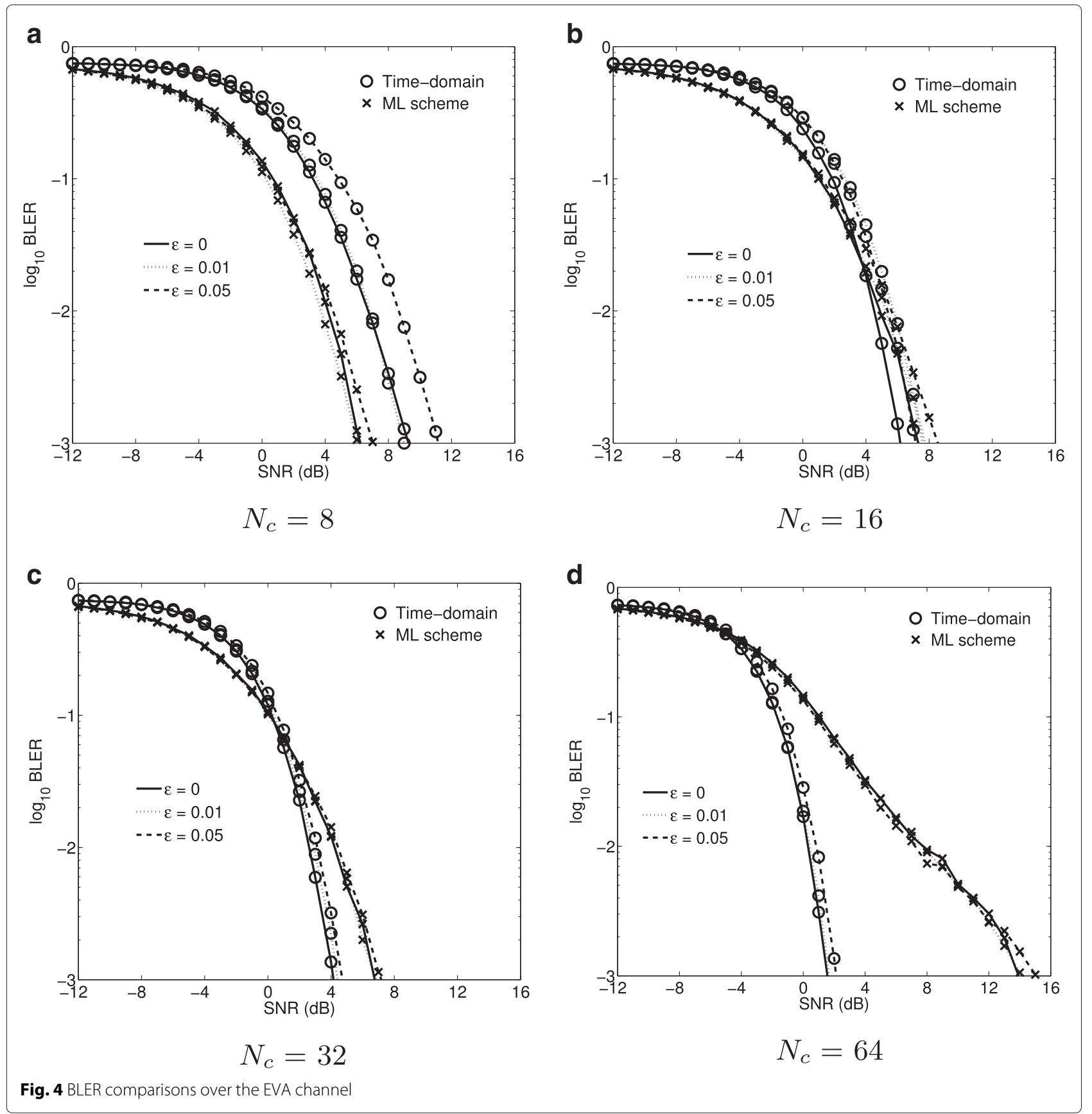

increased. This is because with increased $N_{c}$, more subcarriers may have experienced severe (deep) channel fading, which can lead to noise enhancement and hence poor detection.

From the results in Figs. 3 and 4, Table 3 shows the estimated SNR required to achieve, for example, a BLER level of $1 \%$ (i.e. BLER $=10^{-2}$ ). From Table 3, it can be noted that the time-domain detection method requires relatively smaller SNR levels compared with the ML scheme when
$N_{c} \geq 16$ (in AWGN) and $N_{c} \geq 32$ (in EVA). For instance, in the presence of channel fading and when $\varepsilon=0.05$, the time-domain estimation method requires around 1.3 (when $N_{c}=32$ ) and $6.4 \mathrm{~dB}$ (when $N_{c}=64$ ) less SNR to achieve the same BLER target (of $1 \%$ ) as the ML scheme. The low SNR requirement of the time-domain method therefore makes it a potential and practical technique for detecting deterministic control signals in OFDM systems, particularly when $N_{c}$ is large. 
Table 3 Estimated SNR $(\mathrm{dB})$ requirements of the ML scheme and the time-domain method at BLER $=1 \%$

\begin{tabular}{|c|c|c|c|c|c|c|}
\hline \multirow[t]{2}{*}{ Channel } & \multirow{2}{*}{$\begin{array}{l}\text { Normalised } \\
\mathrm{CFO}, \varepsilon\end{array}$} & \multirow[t]{2}{*}{ Detection schemes } & \multicolumn{4}{|c|}{ SNR (dB) @ BLER=1\% } \\
\hline & & & $N_{c}=8$ & $N_{C}=16$ & $N_{c}=32$ & $N_{C}=64$ \\
\hline \multirow[t]{6}{*}{ AWGN } & \multirow[t]{2}{*}{$\varepsilon=0$} & $M L$ & 2.2 & 1.9 & 1.4 & 0.3 \\
\hline & & Time-domain & 3.9 & 0.5 & -2.5 & -5.1 \\
\hline & \multirow[t]{2}{*}{$\varepsilon=0.01$} & $M L$ & 2.7 & 1.8 & 1.1 & 0.1 \\
\hline & & Time-domain & 3.7 & 0.3 & -2.6 & -5.1 \\
\hline & \multirow[t]{2}{*}{$\varepsilon=0.05$} & $M L$ & 2.5 & 1.9 & 1.4 & 0.4 \\
\hline & & Time-domain & 4.3 & 0.4 & -2.5 & -5.2 \\
\hline \multirow[t]{6}{*}{ EVA } & \multirow[t]{2}{*}{$\varepsilon=0$} & $M L$ & 4.2 & 4.9 & 4.3 & 7.8 \\
\hline & & Time-domain & 6.8 & 4.7 & 2.7 & 0.5 \\
\hline & \multirow[t]{2}{*}{$\varepsilon=0.01$} & $M L$ & 3.8 & 5.3 & 4.3 & 7.7 \\
\hline & & Time-domain & 6.9 & 5.8 & 2.9 & 0.6 \\
\hline & \multirow[t]{2}{*}{$\varepsilon=0.05$} & $M L$ & 4.6 & 5.7 & 4.5 & 7.3 \\
\hline & & Time-domain & 8.7 & 5.5 & 3.2 & 0.9 \\
\hline
\end{tabular}

\section{Conclusions}

This paper considered the detection of deterministic SCI in a baseband OFDM architecture. The detection performance of a time-domain correlation method is investigated and compared against the conventional ML estimation method. A key benefit of the time-domain estimation method is that it requires no channel estimation at the receiver. The detection performance of the timedomain estimation method is found to be largely dependent on the value of $N_{c}$, which represents the size of the $\mathrm{SCI}$. In the presence of channel fading and CFO, the timedomain method achieves an improved detection performance over the ML approach when $N_{c} \geq 32$. Therefore, the time-domain method is a viable and a potential detection scheme for detecting deterministic control information often encountered in practical wireless OFDM systems.

\section{Abbreviations}

AWGN, additive white Gaussian noise; BER, bit error rate; BLER, block error rate; $\mathrm{CFI}$, control format indicator; CFO, carrier frequency offset; $\mathrm{CP}$, cyclic prefix; EVA, extended vehicular type A; FFT, fast Fourier transform; IFFT, inverse fast Fourier transform; ISI, inter-symbol interference; LTE, Long-Term Evolution; ML, maximum likelihood; MMSE, minimum mean square error; OFDM, orthogonal frequency division multiplexing; RMS, root mean square; $\mathrm{SCl}$, selective control information; SNR, signal-to-noise ratio; QAM, quadrature amplitude modulation

\section{Competing interests}

The authors declare that they have no competing interests.

Received: 27 November 2015 Accepted: 7 July 2016

Published online: 19 July 2016

\section{References}

1. G Ku, JM Walsh, Resource allocation and link adaptation in LTE and LTE Advanced: a tutorial. Commun. Surv. Tutor. IEEE. 17(3), 1605-1633 (2015)

2. TVK Chaitanya, EG Larsson, Improving 3GPP-LTE uplink control signaling performance using complex-field coding. Vehicular Technol. IEEE Trans. 62(1), 161-171 (2013)
3. A Damnjanovic, J Montojo, Y Wei, T Ji, T Luo, M Vajapeyam, T Yoo, O Song D Malladi, A survey on 3GPP heterogeneous networks. Wireless Commun. IEEE. 18(3), 10-21 (2011)

4. S Coleri, M Ergen, A Puri, A Bahai, Channel estimation techniques based on pilot arrangement in OFDM systems. Broadcasting, IEEE Trans. 48(3), 223-229 (2002)

5. K Zhong, X Lei, S Li, in High Mobility Wireless Communications (HMWC), 2013 International Workshop On. Channel estimation and data detection for OFDM systems in practical communications environment, (Shanghai, China, 2013), pp. 1-5

6. M Morelli, U Mengali, A comparison of pilot-aided channel estimation methods for OFDM systems. Signal Process. IEEE Trans. 49(12), 3065-3073 (2001)

7. SJ Thiruvengadam, LMA Jalloul, Performance analysis of the 3GPP-LTE physical control channels. EURASIP J. Wireless Commun. Netw. 2010, $1-10(2010)$

8. MO Damen, H El Gamal, G Caire, On maximum-likelihood detection and the search for the closest lattice point. Inform. Theory, IEEE Trans. 49(10), 2389-2402 (2003)

9. K Su, I Berenguer, IJ Wassell, X Wang, Efficient maximum-likelihood decoding of spherical lattice codes. Commun. IEEE Trans. 57(8), 2290-2300 (2009)

10. J Pan, W-K Ma, J Jalden, MIMO Detection by Lagrangian dual maximum-likelihood relaxation: reinterpreting regularized lattice decoding. Signal Process. IEEE Trans. 62(2), 511-524 (2014)

11. V Le Nir, T van Waterschoot, J Duplicy, M Moonen, Blind coarse timing offset estimation for CP-OFDM and ZP-OFDM transmission over frequency selective channels. EURASIP J. Wireless Commun. Netw. 2009, 44 (2009)

12. LB Le, V Lau, E Jorswieck, N-D Dao, A Haghighat, DI Kim, T Le-Ngoc, Enabling $5 \mathrm{G}$ mobile wireless technologies. EURASIP J. Wireless Commun. Netw. 2015(1), 1-14 (2015)

13. NI Miridakis, DD Vergados, A survey on the successive interference cancellation performance for single-antenna and multiple-antenna OFDM systems. Commun. Surveys Tutor. IEEE. 15(1), 312-335 (2013)

14. $3 G P P T S 36211$ v12.0.0, Evolved universal terrestrial radio access (E-UTRA). Physical channels and modulation (2013)

15. MK Ozdemir, H Arslan, Channel estimation for wireless OFDM systems. Commun. Surv. Tutor. IEEE. 9(2), 18-48 (2007)

16. X Cheng, Q Yao, M Wen, CX Wang, LY Song, BL Jiao, Wideband channel modeling and intercarrier interference cancellation for vehicle-to-vehicle communication systems. IEEE J. Selected Areas Commun. 31(9), 434-448 (2013)

17. $M$ Wen, $X$ Cheng, J Wu, L Yang, B Jiao, Optimal correlative coding for discrete-time OFDM systems. IEEE Trans. Vehicular Technol. 63(2), 987-993 (2014) 
18. 3GPP TS 36212 v12.0.0, Evolved universal terrestrial radio access (E-UTRA). Multiplexing and channel coding (2013)

19. L Cimini, Analysis and simulation of a digital mobile channel using orthogonal frequency division multiplexing. Commun. IEEE Trans. 33(7), 665-675 (1985)

20. A Peled, A Ruiz, in Acoustics, Speech, and Signal Processing, IEEE International Conference on ICASSP' 80 . Frequency domain data transmission using reduced computational complexity algorithms, vol. 5, (Denver, USA, 1980), pp. 964-967

21. L Weng, EKS Au, PWC Chan, RD Murch, RS Cheng, WH Mow, VKN Lau, Effect of carrier frequency offset on channel estimation for SISO/MIMO-OFDM systems. Wireless Commun. IEEE Trans. 6(5), 1854-1863 (2007)

22. HC Nguyen, E de Carvalho, R Prasad, Multi-user interference cancellation schemes for carrier frequency offset compensation in uplink OFDMA. Wireless Commun. IEEE Trans. 13(3), 1164-1171 (2014)

23. O Edfors, M Sandell, J-J van de Beek, SK Wilson, PO Borjesson, OFDM channel estimation by singular value decomposition. Commun. IEEE Trans. 46(7), 931-939 (1998)

24. H Zamiri-Jafarian, H Khoshbin, S Pasupathy, Time-domain equalizer for OFDM systems based on SINR maximization. Commun. IEEE Trans. 53(6), 924-929 (2005)

25. J Balakrishnan, RK Martin, CR Johnson, Blind, adaptive channel shortening by sum-squared auto-correlation minimization (SAM). Signal Process. IEEE Trans. 51(12), 3086-3093 (2003)

26. H Minn, VK Bhargava, An investigation into time-domain approach for OFDM channel estimation. Broadcasting, IEEE Trans. 46(4), 240-248 (2000)

27. JM Davis, IA Gravagne, RJ Marks, in SystemTheory (SSST), 2010 42nd Southeastern SymposiumOn. Time scale discrete Fourier transforms, (Texas, USA, 2010), pp. 102-110

28. SA Adegbite, SG McMeekin, BG Stewart, Time-domain Sl estimation for SLM based OFDM systems without SI transmission. Springer Wireless Personal Commun. 85(3), 1193-1203 (2015)

29. 3GPP TS 36101 v12.0.0, Evolved universal terrestrial radio access (E-UTRA); user equipment (UE) radio transmission and reception (2013)

30. S Adegbite, BG Stewart, SG McMeekin, Least squares interpolation methods for LTE system channel estimation over extended ITU channels. Int. J. Inform. Electron. Eng. 3(4), 414-418 (2013)

\section{Submit your manuscript to a SpringerOpen ${ }^{\circ}$ journal and benefit from:}

- Convenient online submission

- Rigorous peer review

- Immediate publication on acceptance

- Open access: articles freely available online

- High visibility within the field

- Retaining the copyright to your article

Submit your next manuscript at $\gg$ springeropen.com 